\title{
Explanation of observational data engenders a causal belief about smoking and cancer
}

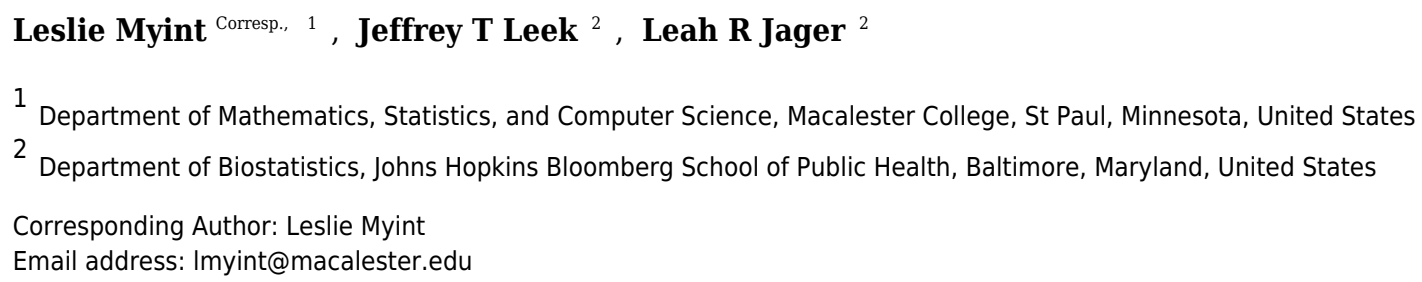

Most researchers do not deliberately claim causal results in an observational study. But do we lead our readers to draw a causal conclusion unintentionally by explaining why significant correlations and relationships may exist? Here we perform a randomized controlled experiment in a massive open online course run in 2013 that teaches data analysis concepts to test the hypothesis that explaining an analysis will lead readers to interpret an inferential analysis as causal. We test this hypothesis with a single example of an observational study on the relationship between smoking and cancer. We show that adding an explanation to the description of an inferential analysis leads to a $15.2 \%$ increase in readers interpreting the analysis as causal (95\% confidence interval for difference in two proportions: $12.8 \%-17.5 \%)$. We then replicate this finding in a second large scale massive open online course. Nearly every scientific study, regardless of the study design, includes an explanation for observed effects. Our results suggest that these explanations may be misleading to the audience of these data analyses and that qualification of explanations could be a useful avenue of exploration in future research to counteract the problem. Our results invite many opportunities for further research to broaden the scope of these findings beyond the single smoking-cancer example examined here. 
1 Title: Explanation of observational data engenders a causal belief about smoking and

2 cancer

3 Authors: Leslie Myint ${ }^{1 *}$, Jeffrey T. Leek², and Leah R. Jager ${ }^{2}$

4

5 1. Department of Mathematics, Statistics, and Computer Science. Macalester College

6 2. Department of Biostatistics. Johns Hopkins Bloomberg School of Public Health

7 * To whom correspondence should be addressed: 1myint@macalester.edu

ABSTRACT

Most researchers do not deliberately claim causal results in an observational study. But do we

lead our readers to draw a causal conclusion unintentionally by explaining why significant correlations and relationships may exist? Here we perform a randomized controlled experiment in a massive open online course run in 2013 that teaches data analysis concepts to test the hypothesis that explaining an analysis will lead readers to interpret an inferential analysis as causal. We test this hypothesis with a single example of an observational study on the relationship between smoking and cancer. We show that adding an explanation to the description of an inferential analysis leads to a $15.2 \%$ increase in readers interpreting the analysis as causal (95\% confidence interval for difference in two proportions: $12.8 \%-17.5 \%)$. We then replicate this finding in a second large scale massive open online course. Nearly every scientific study, regardless of the study design, includes an explanation for observed effects. Our results suggest that these explanations may be misleading to the audience of these data analyses and that qualification of explanations could be a useful avenue of exploration in future research to counteract the problem. Our results invite many opportunities for further research to broaden the scope of these findings beyond the single smoking-cancer example examined here. 


\section{ARTICLE}

28

29

30

Drinking too much tea causes prostate cancer (PTI, 2016), eating chocolate helps people stay thin (Jaslow, 2012), junk food during pregnancy leaves children fat for life (Mirror.co.uk, 2008). We all know that correlation does not imply causation, but we have also all seen exaggerated headlines in the media that fall short in capturing the true results of a scientific study. A recent report in the British Medical Journal found the fault may not lie entirely with the media (Sumner et al., 2014), but may be aided by exaggerated press releases from universities themselves. In fact, in their study of 462 press releases, the study authors found that 33\% (95\% confidence interval: $26 \%$ to $40 \%$ ) contained exaggerated causal claims. Regardless, of where the exaggeration happens, a result seems more realistic if you can explain why you think it is happening.

Most researchers do not deliberately claim causal results in an observational study. But do we lead our readers to draw a causal conclusion unintentionally by explaining why significant correlations and relationships may exist? Once we discover that an association exists, it is natural to want to explain why it does. We may describe potential mechanisms, make connections to previous literature, or put an observation in context. Despite these explanations, causal relationships are not proven in a single observational study and are only increasingly substantiated over the course of many such studies. There is observational evidence suggesting a noticeable prevalence of inappropriate causal language in both nutritional (Cofield, Corona \& Allison, 2010) and educational (Robinson et al., 2007) research studies. 
50 The distinction between correlational and causal evidence is not merely a pedantic formality.

51 Because causal statements carry moral underpinnings, they can have dangerous consequences for

52 societal perceptions of certain groups, products, or practices when consumed and interpreted by

53 the general public (Lombrozo, 2017). For example, researchers of developmental origins of

54 health and disease published a cautionary commentary in response to a collection of headlines

55 (Mother's diet during pregnancy alters baby's DNA, Pregnant 9/11 survivors transmitted trauma

56 to their children) that seemed to vilify mothers for developmental outcomes in babies

57 (Richardson et al., 2014). In research areas dealing with human subjects, mistakes in perceptions

58 about evidence can be harmful, and reporters must use great care in the language they use to

59 describe scientific findings. The danger in these headlines and in related causal language (e.g.

60 explanatory statements, jargon) lies not in the words themselves but in their interpretation by the 61 public.

62

63 In this work, we investigate how interpretation of scientific evidence is affected by a specific

64 area of causal language: explanation. We report the results of a randomized experiment

65 performed on an online educational platform that suggest a strong effect of explanatory language

66 on students' perception of whether a study is correlational or causal. We emphasize that the

67 results presented here pertain to a single example (an observational smoking-cancer study) and

68 that there are many opportunities for further research to clarify the extent of generalizability of

69 these results.

70

71

72 METHODS

73 
74 Different types of studies have different analysis goals (Table 1) (Leek \& Peng, 2015). We were

75 interested in whether people can distinguish between a study whose goal was inferential and one

76 whose goal was actually causal, as this is a common error often termed "correlation does not

77 equal causation". We wanted to know whether including language explaining an observed

78 association leads people to believe that an inferential study is causal. To test this hypothesis, we

79 considered an experiment in a large online open-access data analysis course, for which one of the

80 authors was an instructor. The other two authors had no relationship with the online course, and

81 none of the authors had access to the participants' identities. This introductory-level course

82 covered basic data analytic concepts. Our experiment involved a single randomized quiz

83 question administered during the course. This experiment originally ran in January 2013 and was

84 later independently replicated in a separate offering of the course in October 2013. Between

85 these two replications, over 22,000 students completed versions of our experimental question.

86 These experimental questions were initially included as part of a regular quiz within the course;

87 as such, we did not prospectively obtain informed consent. We later sought and were granted

88 IRB approval to perform the analyses presented here (Johns Hopkins Bloomberg School of

89 Public Health IRB number 00005988.) Consent was waived on the grounds that this

90 retrospective analysis of the data was of a low-risk educational type and that we would remove

91 any individualized identifiers in the data.

92

93 Early in the course, students were presented with the definitions of six possible types of data

94 analysis (descriptive, exploratory, inferential, predictive, causal, and mechanistic) consistent with

95 those shown in Table 1. In the subsequent course quiz, we provided students with a description

96 of an inferential study - from which we can only infer correlation: 
97

98

99

100

101

102

103

104 105

106

107

108

109

110

111

112

113

114

115

116

117

118

119
We take a random sample of individuals in a population and identify whether they smoke and if they have cancer. We observe that there is a strong relationship between whether a person in the sample smoked or not and whether they have lung cancer. We claim that the smoking is related to lung cancer in the larger population.

We randomized students to see or not see an explanatory interpretation accompanying this description. Students in this explanatory interpretation group saw an additional sentence:

.

We explain we think that the reason for this relationship is because cigarette smoke contains known carcinogens such as arsenic and benzene, which make cells in the lungs become cancerous.

All students were then asked to identify the type of analysis for these results. In addition to the correct answer (inferential), students were presented at random with three of four possible incorrect answer choices (descriptive, causal, predictive, mechanistic). That is, approximately $25 \%$ of students made their choice from inferential, descriptive, causal, and predictive, approximately $25 \%$ from inferential, descriptive, causal, and mechanistic, and so on. That only four answer choices could be shown at the same time was a limitation of the online platform. Although the described analysis is inferential in nature, we hypothesized that students who saw the explanatory language would be more likely to identify the analysis as causal if given that choice. Because students were able to retake this quiz multiple times in order to achieve a passing grade, we collected answers from each student's first attempt. We compared the 
120 proportions of the causal and inferential answer choices between the explanatory language and

121 non-explanatory language arms using two sample tests for differences in proportions (Table 2).

122

123

124

125

126

127

128

129

130

131

132

133

134

135

136

137

138

139

140

141

142

143

144 causal.

\section{RESULTS}

In our original experiment (January 2013), 20,257 students completed our experimental quiz question. These students were randomly assigned to one of four arms, where each arm contained the correct answer choice (inferential) and three incorrect answer choices (from among causal, descriptive, predictive, and mechanistic). Sample sizes are given in Table 2. We present detailed results for two arms: (1) students who chose between inferential, causal, predictive, and mechanistic analyses and (2) students who were not given causal as a choice, but instead chose between inferential, descriptive, predictive, and mechanistic analyses. Detailed results for the other student groups can be found in the Supplementary Information. Table 2 shows summary results for the four groups of students corresponding to the four sets of answer choices seen.

Among students selecting from inferential, causal, predictive, and mechanistic answer choices, the majority (68.5\%) correctly answered that the description referred to an inferential data analysis (Table 3). However, a significantly higher percentage of students who were shown the explanatory language claimed it was a causal analysis compared to students who did not see the additional language: $31.8 \%$ compared to $16.6 \%$ (Difference in proportions: $15.2 \% .95 \%$ CI for difference in two proportions: $12.8 \%-17.5 \%$ ) (Table 2). These results indicate that explanatory language increases the chance a student will mistake an inferential result as causal. In this case, students who saw the additional explanation were almost twice as likely to claim the results as

145 
146 This increase in the choice of a causal analysis when faced with explanatory language

147 corresponded to a decrease in choice of an inferential analysis. The percentages of students who

148 chose either a predictive or descriptive analysis were similar between the two treatment groups.

149 However, there was an increase in the percentage of students who claimed the result was

150 mechanistic in the explanatory language group: 3.5\% compared to $1.2 \%$ (Difference in

151 proportions: $2.3 \%$. 95\% CI for difference in two proportions: $1.5 \%-3.2 \%$ ). This is not surprising

152 since a mechanistic result is similar to a causal result in that it describes a deterministic process

153 by which one variable affects another.

155 Among students who were not given the option to select "causal" as an answer (selecting instead 156 from inferential, predictive, descriptive, and mechanistic analyses), a higher percentage (84.6\%) 157 correctly answered that the description referred to an inferential data analysis (Table 4). In this 158 case, a significantly higher percentage of students correctly claimed the analysis was inferential 159 when not shown the explanatory language: $88.2 \%$ compared to $80.9 \%$ (Difference in 160 proportions: 7.3\%. 95\% CI for difference in two proportions: 5.2\% - 9.3\%) (Table 2). These 161 results indicate that, even without the ability to identify the analysis as causal, students had a 162 harder time correctly identifying an inferential study when given hypothesized information about 163 the reason for a correlation. The size of the effect is much smaller than with the causal answer 164 option, however. The decrease in correct answers again corresponded to an increase in choice of 165 a mechanistic analysis: 5.6\% compared to $1.4 \%$ (Difference in proportions: $4.2 \% .95 \%$ CI for 166 difference in two proportions: $3.2 \%-5.3 \%$ ).

167 168 169
To confirm our results, we analyzed data from an independent replication of our experiment in a later offering of the same data analysis course. In the replication (October 2013), 1,762 students 
170 completed our experimental quiz question. The results of this replication were consistent with

171 those in the original experiment (Table 2, 3, 4, and Supplementary Information). Differences in

172 percentages for the causal and inferential answer choices were always of the same sign between

173 the two courses, and the magnitudes of the differences were also similar (Table 2). While the

174 sample size in this course is much smaller, the concordance of results and the maintenance of

175 experimental procedures between courses align with a statistical definition of replicability that

176 has been put forth (Patil, Peng \& Leek, 2016).

177

178

179

180

181

182

183

184

185

186

187

188

189

190

191

192

193

194

\section{CONCLUSIONS}

We know that the way data is visualized can affect how well people derive information from graphs (Cleveland \& McGill, 1985). The results of this experiment suggest that the way we write about a data analysis may also be critical. By performing a randomized controlled trial, we have shown clear evidence of an effect of explanatory statements on perceptions of research results for a specific scenario and replicated this effect in a second experiment. The nature of our study design justifies the use of causal language to describe the precise effect of explanatory language on categorical perceptions of research findings, but it is important to keep in mind that these effects are specific to a certain population of learners and to our specific quiz question. Further, our study does not elucidate any of the latent psychological or cognitive mechanisms that give rise to the shift in categorical perceptions. In the remainder of this section, we discuss these limitations and avenues for further research.

One limitation of our study is the population of participants. We performed this randomized trial in a study population of learners in a massive open online course (MOOC) as opposed to a 
195 representative sample of the target population of English speakers. We do not have access to 196 demographic information on the learners in our trial because the online platform on which we 197 conducted this study did not collect such information at the time. The composition of students in 198 MOOCs has changed rapidly since then, so we are also unable infer demographics confidently. 199 Some general research into MOOC demographics has been conducted; surveys indicate that 200 these learners are slightly more likely to be male, often have bachelor's degrees, and typically 201 have some level of employment (Bayeck, 2016). Learners in these online courses report a variety 202 of motivations for taking the courses, suggesting at least some lifestyle diversity. However, there 203 is certainly a gap between our MOOC study population and our target population, and we 204 advocate rectifying this gap with collection of pertinent demographic information in future 205 studies.

206

207 A second limitation of our study was the choice to use smoking and cancer as the scenario in our 208 quiz question. Belief bias describes the tendency of individuals to judge the strength of a 209 statistical claim based on its plausibility rather than on the data and methods themselves, and it is 210 likely to be operating in this study. The causal link between smoking and lung cancer has been 211 firmly established over time through an accumulation of evidence, so although the wording of 212 our quiz question does not describe a causal study, belief bias likely nudges learners to think 213 otherwise. Had we used a different example, the effect of the explanatory text would likely have 214 been smaller. We urge future studies to use examples that span a wider range of evidence for a 215 causal relationship (e.g. the smoking example used here and one involving the link between yoga 216 and anxiety disorders). As we discuss later, this will be key to improving our understanding 217 about when exactly explanation contributes to causal misinterpretations. 
219 There are specific avenues of further research that would elucidate the situations in which the 220 presence of explanation influences the formulation of causal interpretations. As we have just 221 discussed, the example of smoking and cancer is a difficult one for learners to contend with 222 because the causal relationship has actually very well substantiated over time. Further the 223 wording of the explanatory text is pretty forceful in that it describes arsenic and benzene as being 224 "known carcinogens, which make cells in the lungs become cancerous". Despite the strong 225 wording of the statement, it is still unclear if the amount of these compounds in cigarettes is 226 sufficient to be carcinogenic. This is a subtle point and further increases the difficulty of this 227 question for students who are randomized to see the explanation. Future experiments should vary 228 the persuasiveness of the explanatory text from being hypothetical but convincing to being far229 fetched. The degree of persuasiveness of the explanatory text and additional nuances of how the 230 explanation is conveyed might have an impact on specific cognitive mechanisms leading to 231 causal interpretation. These additional nuances might include providing citations for claims, 232 varying the level of detail in the explanation, or making known additional explanations that can 233 be substantiated or discredited. If future experiments reveal differences in these modes of 234 presentation, we could much better understand why misinterpretations occur.

236 Another limitation that is somewhat orthogonal to our use of specific features in the explanation 237 text is the span of the scientific domain covered by our quiz question. The particular example we 238 used falls within the field of medical biology. Future experiments should represent a diverse set 239 of fields including social science, political science, economics, medicine, and biology subfields. 240 It is possible that explanations with the same features carry different weight across fields. For 
241 example, it could be that biological explanations have greater cognitive impact because they are

242 rooted in physical principles. Experiments that cover interactions between the field explored and

243 the explanation provided have the potential to inform specific guidelines for reporting in

244 different domains.

245

246 Our study has focused on the nature of causal misinterpretation in the presence of explanatory

247 language. We have not investigated any strategies for combating these misinterpretations, an area

248 of future research that could lead to actionable interventions regarding scientific communication.

249 We recognize that it is quite difficult to avoid any explanation when communicating scientific

250 results because explanation is a key means of interpreting research findings. Interpretation is

251 essential for combining different sources of information and advancing our understanding. In

252 both academic and mainstream scientific writing, there is a desire to put results into context,

253 including hypothesized mechanistic explanations to enhance the narrative around a set of

254 empirical results. Nearly every study includes this type of explanation in the discussion section.

255 However, our results suggest that such efforts may actually cause a certain population of readers

256 to be misled about the strength of the scientific evidence. The misinterpretation may be

257 exacerbated by the phenomenon that readers are swayed to believe a statement when they are

258 told scientists understand it (Sloman \& Rabb, 2016). Because interpretation, and thus

259 explanation, is an essential aspect of science communication, we should not aim to avoid

260 explanation but to understand how certain characteristics of explanation help or hinder

261 perception. We hypothesize that it may be beneficial for readers' perceptions to follow up any

262 explanations with warnings against interpreting results causally. Further research is needed to

263 determine if this could counteract the effect of explanations on causal perceptions. 
265 Because our study explores the nature of causal misinterpretation, it is also worth discussing

266

267 268 269 270

271

272 273 274 275 276

277 278 279 280 281 282 283 prior beliefs are derived.

285 A second reason for our discomfort with the causal categorization of the smoking-cancer 286 analysis is that we have a certain definition of causality in mind that the quiz question text 
287 simply does not support. The definition of a causal analysis presented in Table 1 describes the 288 goal as describing (quantifying) how one variable changes on average when another variable is 289 changed. Implicit in this definition is the ability to manipulate the intervention variable, and this 290 ability to manipulate is not an absolute necessity in other forms of causality that have been 291 discussed in other areas, such as the social sciences. Also implicit in this definition is the idea of 292 ceteris paribus; all other variables remaining equal, what is the effect of manipulating the 293 intervention variable, on average? For both of these reasons, it is important for further research 294 to dissect more precisely the understanding of the word "cause" that is triggered when students 295 see explanation accompanying the presentation of a statistical association. In the social sciences, 296 there is a concept of sufficient motive causality that describes how belief in a causal relationship 297 can be substantiated by explaining why a sufficient set of motives could have led to a result 298 (Tellings 2017). For example, this type of reasoning is used by historians to understand why 299 certain events occurred. Although sufficient motive causality is at odds with the definition we 300 have used in this study, we do see a reconciliation. Sufficient motive causality is used in the 301 social sciences because practitioners can assess the quality of evidence that it provides and 302 because awareness of sufficient motives for an effect is sometimes all that is needed to elicit 303 some other desired action. Both of these reasons are quite justifiable in that both acknowledge 304 the role of explanation in causal reasoning as the Bradford Hill criteria also do, but they also 305 make clear the consequences of buying in to such thinking. Assessing the quality of a sufficient 306 motive explanation makes clear that an explanation for a result does not provide guaranteed 307 actionable answers as if through a clear physical mechanism, a misconception known as 308 statistical reification (Greenland 2017). In assessing the quality of an explanation, practitioners 309 should be mindful of various factors that can influence their judgment: strength of prior beliefs, 
310 populations for which the explanation holds, and details of statistical methods used to generate

311 the explanation (Colombo, Bucher \& Sprenger, 2017). If an individual consciously thinks about

312 these matters, there is at least a healthy degree of transparency about the explanation. Using

313 explanations to motivate desired actions also involves transparency regarding the nature of the

314 explanation. For example, if a therapist knows that a treatment regimen works for her patient but

315 not how, it is still worthwhile to convey an explanation for how the treatment works to motivate

316 her patient to continue being treated. In a sense, the construction of this explanation is a causal

317 analysis of the situation.

318

319 It could be that students in this study are increasingly categorizing the smoking-cancer study as a

320 causal analysis in the presence of explanation because they are using reasoning similar to

321 sufficient motive causality as described above. In this case they are perhaps not wrong in the

322 grander debate about defining causal analysis. However we still have concerns that relate to the

323 second implicit part of our definition of a causal analysis: the idea of ceteris paribus. Comparing

324 individuals who smoke to those who do not smoke, who are otherwise identical, is there truly an

325 increase in the proportion who develop lung cancer? Our worry is that explanation that

326 accompanies an inferential result might lead individuals to forget this idea and not think carefully

327 about confounding. Future experiments should carefully use wording to elicit specific concepts

328 of causality such that participant responses more directly indicate thought processes.

329

330 In conclusion, we have shown a clear effect of explanations on categorical perceptions of

331 statistical results in a specific scenario. However, it remains unclear what this implies about

332 cognitive processes or actions that may result from interpretations. In the discussion above, we 
333 have highlighted specific avenues of further research that would better elucidate when, why, and

334 how changes in interpretation follow from explanatory language. These avenues include

335 broadening the populations studied, diversifying the subject domains covered, varying features

336 of the explanatory language, and isolating different components of the definitions of causality to

337 understand subsequent intentions. The last of these will be the most difficult but arguably the

338 most important because it could shed light on what people might actually do in response to

339 consuming a statistical result with explanation, whatever the semantics of causation might be.

340 For this, allowing more flexibility in responses (e.g. free text) might better facilitate capturing

341 these thoughts. With future research, we hope that the community can reach a level of

342 understanding regarding the effects of explanation that engenders vastly improved scientific

343 communication.

344

345

346 The code and data used to perform this analysis are available at:

347 https://github.com/leekgroup/explanatory language 


\section{REFERENCES}

349 Bayeck RY. 2016. Exploratory study of MOOC learners' demographics and motivation: The 350 case of students involved in groups. Open Praxis 8:223-233. DOI:

$351 \quad$ 10.5944/openpraxis.8.3.282.

352 Cleveland WS., McGill R. 1985. Graphical perception and graphical methods for analyzing $353 \quad$ scientific data. Science 229:828-833. DOI: $10.1126 /$ science.229.4716.828.

354 Cofield SS., Corona RV., Allison DB. 2010. Use of causal language in observational studies of 355 obesity and nutrition. Obesity facts 3:353-356. DOI: 10.1159/000322940.

356 Colombo M., Bucher L., Sprenger J. 2017. Determinants of Judgments of Explanatory Power: 357 Credibility, Generality, and Statistical Relevance. Frontiers in psychology 8:1430. DOI: $358 \quad$ 10.3389/fpsyg.2017.01430.

359 Greenland S. 2017. Invited Commentary: The Need for Cognitive Science in Methodology. 360 American Journal of Epidemiology 186 (6): 639-45. DOI: 10.1093/aje/kwx259.

361 Höfler M. 2005. The Bradford Hill Considerations on Causality: A Counterfactual Perspective.

362 Emerging Themes in Epidemiology 2 (November): 11. DOI: 10.1186/1742-7622-2-11.

363 Jaslow R. 2012. Eating lots of chocolate helps people stay thin, study finds. Available at 364 http://www.cbsnews.com/news/eating-lots-of-chocolate-helps-people-stay-thin-study-finds/ $365 \quad$ (accessed May 31, 2017).

366 Leek BJ., Peng RD. 2015. What is the question? Science. DOI: 10.1126/science.aaa6146.

367 Lombrozo T. 2017. The Dangers Of Hidden Jargon In Communicating Science. Available at 368 https://www.npr.org/sections/13.7/2017/06/12/532554252/the-dangers-of-hidden-jargon-in369 communicating-science (accessed January 18, 2018).

370 Mirror.co.uk. 2008. “Junk Food in Pregnancy Leaves Children Fat for Life.” Mirror. June 30, 

children-316010 (accessed June 24, 2018).

373 Mohr SB., Gorham ED, Alcaraz JE, Kane CI, Macera CA, Parsons JK, Wingard DL, and 374 Garland CF. 2012. "Does the Evidence for an Inverse Relationship between Serum Vitamin 375 D Status and Breast Cancer Risk Satisfy the Hill Criteria?” Dermato-Endocrinology 4 (2): 376 152-57. DOI: $10.4161 /$ derm.20449.

377 Patil P., Peng RD., Leek J. 2016. A statistical definition for reproducibility and replicability. 378 bioRxiv:066803. DOI: 10.1101/066803.

379 PTI. 2016.Drinking too much tea can cause prostate cancer: study. Available at 380 http://www.thehindu.com/sci-tech/health/medicine-and-research/drinking-too-much-teacan-cause-prostate-cancer-study/article3547285.ece (accessed November 13, 2017).

Rasmussen SA, Jamieson DJ, Honein MA, and Petersen LR. 2016. “Zika Virus and Birth Defects--Reviewing the Evidence for Causality." The New England Journal of Medicine 374 (20): 1981-87. DOI: 10.1056/NEJMsr1604338.

Richardson SS., Daniels CR., Gillman MW., Golden J., Kukla R., Kuzawa C., Rich-Edwards J. 2014. Society: Don't blame the mothers. Nature 512:131-132. DOI: 10.1038/512131a.

Robinson DH., Levin JR., Thomas GD., Pituch KA., Vaughn S. 2007. The Incidence of “Causal” Statements in Teaching-and-Learning Research Journals. American educational research journal 44:400-413. DOI: 10.3102/0002831207302174.

Sloman SA., Rabb N. 2016. Your Understanding Is My Understanding: Evidence for a Community of Knowledge. Psychological science 27:1451-1460. DOI: 10.1177/0956797616662271.

Sumner P., Vivian-Griffiths S., Boivin J., Williams A., Venetis CA., Davies A., Ogden J., 
394 Whelan L., Hughes B., Dalton B., Boy F., Chambers CD. 2014. The association between 395 exaggeration in health related science news and academic press releases: retrospective 396 observational study. BMJ 349:g7015. DOI: 10.1136/bmj.g7015.

397 Tellings A. 2017. "Evidence-Based Practice in the Social Sciences? A Scale of Causality, 398 Interventions, and Possibilities for Scientific Proof." Theory \& Psychology 27 (5): 581-99. 399 DOI: $10.1177 / 0959354317726876$. 


\section{Table $\mathbf{1}$ (on next page)}

Goals for different analysis types (Leek \& Peng, 2015).

These analysis types form the set of possible answer choices in our randomized experiment and were taught to students before the experiment was performed. 
1 Table 1: Goals for different analysis types (Leek \& Peng, 2015). These analysis types form the set of 2 possible answer choices in our randomized experiment and were taught to students before the experiment 3 was performed.

\begin{tabular}{|l|l|}
\hline \multicolumn{1}{|c|}{ Type of analysis } & \multicolumn{1}{c|}{ Goal of analysis } \\
\hline Descriptive & Summarizing the data without interpretation \\
\hline Exploratory & $\begin{array}{l}\text { Summarizing the data with interpretation, but without generalization beyond } \\
\text { the original sample }\end{array}$ \\
\hline Inferential & $\begin{array}{l}\text { Generalizing beyond the original sample, with the goal of describing an } \\
\text { association in a larger population }\end{array}$ \\
\hline Predictive & $\begin{array}{l}\text { Generalizing beyond the original sample, with the goal of predicting a } \\
\text { measurement for a new individual }\end{array}$ \\
\hline Causal & $\begin{array}{l}\text { Generalizing beyond the original sample, with the goal of learning how } \\
\text { changing the average of one measurement affects, on average, another } \\
\text { measurement }\end{array}$ \\
\hline Mechanistic & $\begin{array}{l}\text { Generalizing beyond the original sample, with the goal of learning how } \\
\text { changing one measurement deterministically affects another variable's } \\
\text { measurement. }\end{array}$ \\
\hline
\end{tabular}

4 


\section{Table 2 (on next page)}

Effect of explanatory language on student responses.

For each of four sets of answer choices seen, differences in the percentage choosing the "causal" and "inferential" answer choices are given, as well as 95\% confidence intervals for the differences and sample sizes. 
1 Table 2: Effect of explanatory language on student responses. For each of four sets of answer choices 2 seen, differences in the percentage choosing the "causal" and "inferential" answer choices are given, as 3 well as $95 \%$ confidence intervals for the differences and sample sizes.

4

\begin{tabular}{|c|c|c|}
\hline \multirow[b]{2}{*}{ Answer choices seen } & \multicolumn{2}{|c|}{$\begin{array}{l}\text { Difference in percentage choosing "causal" when seeing explanatory } \\
\text { language vs. not seeing explanatory language }(95 \% \text { CI for difference in } \\
\text { proportions) }\end{array}$} \\
\hline & January 2013 course & October 2013 course \\
\hline $\begin{array}{l}\text { inferential, causal, descriptive, } \\
\text { predictive }\end{array}$ & $\begin{array}{l}14.5 \%(12.2 \%, 16.8 \%) \\
\mathrm{N}=5061\end{array}$ & $\begin{array}{l}14.3 \%(6.4 \%, 22.2 \%) \\
\mathrm{N}=447\end{array}$ \\
\hline $\begin{array}{l}\text { inferential, causal, descriptive, } \\
\text { mechanistic }\end{array}$ & $\begin{array}{l}15.8 \%(13.4 \%, 18.1 \%) \\
\mathrm{N}=5092\end{array}$ & $\begin{array}{l}14.8 \%(6.6 \%, 23.0 \%) \\
\mathrm{N}=463\end{array}$ \\
\hline \multirow[t]{2}{*}{$\begin{array}{l}\text { inferential, causal, predictive, } \\
\text { mechanistic }\end{array}$} & $\begin{array}{l}15.2 \%(12.8 \%, 17.5 \%) \\
\mathrm{N}=5088\end{array}$ & $\begin{array}{l}19.9 \%(11.5 \%, 28.3 \%) \\
\mathrm{N}=437\end{array}$ \\
\hline & \multicolumn{2}{|c|}{$\begin{array}{l}\text { Difference in percentage choosing "inferential" when seeing explanatory } \\
\text { language vs. not seeing explanatory language ( } 95 \% \text { CI for difference in } \\
\text { proportions) }\end{array}$} \\
\hline $\begin{array}{l}\text { inferential, descriptive, predictive, } \\
\text { mechanistic }\end{array}$ & $\begin{array}{l}-7.3 \%(-9.3 \%,-5.2 \%) \\
\mathrm{N}=5016\end{array}$ & $\begin{array}{l}-4.9 \%(-12.6 \%, 2.9 \%) \\
\mathrm{N}=416\end{array}$ \\
\hline
\end{tabular}




\section{Table 3(on next page)}

Detailed results for the experimental arm with answer choices: inferential, causal, predictive, and mechanistic.

In the presence of explanatory language, nearly twice as many students incorrectly selected "causal" with a corresponding decrease in the percentage of students correctly selecting "inferential”. 
1 Table 3: Detailed results for the experimental arm with answer choices: inferential, causal,

2 predictive, and mechanistic. In the presence of explanatory language, nearly twice as many students

3 incorrectly selected "causal" with a corresponding decrease in the percentage of students correctly

4 selecting "inferential".

\begin{tabular}{|c|c|c|c|c|}
\hline \multirow[b]{2}{*}{$\begin{array}{r}\text { This is an example of a/an } \\
\text { data analysis. }\end{array}$} & \multicolumn{2}{|c|}{$\begin{array}{c}\text { January } 2013 \text { course } \\
(\mathrm{N}=5088)\end{array}$} & \multicolumn{2}{|c|}{$\begin{array}{l}\text { October } 2013 \text { course } \\
\qquad(\mathrm{N}=437)\end{array}$} \\
\hline & $\begin{array}{l}\text { Saw explanatory } \\
\text { language } \\
(\mathrm{N}=2516)\end{array}$ & $\begin{array}{l}\text { No explanatory } \\
\text { language } \\
(\mathrm{N}=2572)\end{array}$ & $\begin{array}{l}\text { Saw explanatory } \\
\text { language } \\
(\mathrm{N}=199)\end{array}$ & $\begin{array}{l}\text { No explanatory } \\
\text { language } \\
(\mathrm{N}=238)\end{array}$ \\
\hline inferential & $1508(59.9 \%)$ & $1977(76.9 \%)$ & $116(58.3 \%)$ & $190(79.8 \%)$ \\
\hline causal & $799(31.8 \%)$ & $427(16.6 \%)$ & $68(34.2 \%)$ & $34(14.3 \%)$ \\
\hline predictive & $120(4.8 \%)$ & $138(5.4 \%)$ & $8(4.0 \%)$ & $11(4.6 \%)$ \\
\hline mechanistic & $89(3.5 \%)$ & $30(1.2 \%)$ & $7(3.5 \%)$ & $3(1.3 \%)$ \\
\hline
\end{tabular}

5 


\section{Table 4 (on next page)}

Detailed results for the experimental arm with answer choices: inferential, descriptive, predictive, and mechanistic (no causal).

In the presence of explanatory language, a lower percentage of students correctly selected "inferential", and a higher percentage of students incorrectly selected "mechanistic". 
1 Table 4: Detailed results for the experimental arm with answer choices: inferential, descriptive,

2 predictive, and mechanistic (no causal). In the presence of explanatory language, a lower percentage of

3 students correctly selected "inferential", and a higher percentage of students incorrectly selected

4 "mechanistic".

\begin{tabular}{|c|cc|cc|}
\hline & \multicolumn{2}{|c|}{$\begin{array}{c}\text { January } 2013 \text { course } \\
(\mathrm{N}=5016)\end{array}$} & \multicolumn{2}{c|}{$\begin{array}{c}\text { October 2013 course } \\
(\mathrm{N}=416)\end{array}$} \\
\cline { 2 - 5 } $\begin{array}{c}\text { This is an example of a/an } \\
\text { data analysis. }\end{array}$ & $\begin{array}{c}\text { Saw explanatory } \\
\text { language } \\
(\mathrm{N}=2485)\end{array}$ & $\begin{array}{c}\text { No explanatory } \\
\text { language } \\
(\mathrm{N}=2531)\end{array}$ & $\begin{array}{c}\text { Saw explanatory } \\
\text { language } \\
(\mathrm{N}=199)\end{array}$ & $\begin{array}{c}\text { No explanatory } \\
\text { language } \\
(\mathrm{N}=217)\end{array}$ \\
\hline inferential & $2011(80.9 \%)$ & $2232(88.2 \%)$ & $160(80.4 \%)$ & $185(85.3 \%)$ \\
predictive & $196(7.9 \%)$ & $181(7.2 \%)$ & $10(5.0 \%)$ & $14(6.5 \%)$ \\
descriptive & $138(5.6 \%)$ & $82(3.2 \%)$ & $14(7.0 \%)$ & $6(2.8 \%)$ \\
mechanistic & $140(5.6 \%)$ & $36(1.4 \%)$ & $15(7.5 \%)$ & $12 \%)$ \\
\hline
\end{tabular}

5 